\title{
Clinical Guideline Highlights for the Hospitalist: The Use of Intravenous Fluids in the Hospitalized Adult
}

\author{
Emily Gottenborg, MD*; Read Pierce, MD
}

'Division of Hospital Medicine, University of Colorado Anschutz Medical Campus, Denver, Colorado.

GUIDELINE TITLE: Intravenous Fluid Therapy in Adults in Hospital

RELEASE DATE: December, 2013

PRIOR VERSION: Not Applicable

DEVELOPER: Multidisciplinary Guideline Development Group within the United Kingdom's National Clinical
Guideline Centre

FUNDING SOURCE: National Institute for Health and Care Excellence

TARGET POPULATION: Hospitalized adult patients

Journal of Hospital Medicine 2019;14:172-173. () 2019 Society of Hospital Medicine

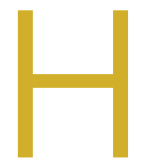
ospitalized patients often receive intravenous fluids (IVF) when they cannot meet physiologic needs through oral intake in the setting of medical or surgical illness. Prescribing the optimal IVF solution to the appropriate patient is a complex decision and often occurs without the same degree of institutionalized restrictions or guidance developed for other inpatient pharmacologic agents. There is wide variation in clinical utilization of IVF due to the lack of data to guide decision making. ${ }^{1}$ When data do exist, they typically focus on a limited number of clinical situations. ${ }^{2}$ Thus, even though IVF are often considered low-risk, the frequency and lack of consistency with which they are used can result in errors, complications, and over-use of medical resources. ${ }^{3}$

\section{KEY RECOMMENDATIONS FOR THE HOSPITALIST}

(Evidence quality: not described in the guideline, recommendation strength: not described in the guideline)

\section{Recommendation 1}

To aid in fluid management and avoid complications, the guidelines recommend that patients on IVF require careful assessment of volume status, including a detailed history, physical exam, clinical monitoring, and daily labs. ${ }^{2}$

Clinical history should focus on understanding fluid losses and intake; physical exam should include vital signs, evidence of orthostatic hypotension, capillary refill, jugular venous pul-

\footnotetext{
*Corresponding Author: Emily Gottenborg, MD; E-mail: emily.gottenborg@ ucdenver.edu; Telephone: 720-848-4289
}

Received: January 25, 2019; Revised: January 30, 2019.

Accepted: January 30, 2019

๑ 2019 Society of Hospital Medicine DOI 10.12788/jhm.3178 sation, and assessment for pulmonary edema. Subsequent clinical monitoring should include fluid balance (Ins and Outs) and daily weights. All patients starting or continuing IVF should have a basic metabolic panel at least daily according to the guidelines, though the authors note this frequency may be too high for some patients and needs further study. ${ }^{2}$

\section{Recommendation 2}

The guidelines describe four types of IV fluids that can be administered: crystalloids, balanced crystalloids, glucose solutions, and non blood-product colloids. ${ }^{2}$

Crystalloids include isotonic saline with 154 millimoles (mmol) of sodium and chloride. Balanced crystalloids, such as lactated Ringer's solution, are more physiologic, with less sodium and chloride, and the addition of magnesium, potassium, and calcium. Glucose solutions are quickly metabolized and, thus, are an effective way to deliver free water. Non blood-product colloids include particles that are retained within the circulation, including proteins such as human albumin.

\section{Recommendation 3}

For each indication to administer IVF, the guidelines recommend the following formulations and considerations: ${ }^{2}$

For general resuscitation, use crystalloids with sodium content of 130-154 mmol, delivered in a bolus of at least 500 milliliters $(\mathrm{mL})$ over 15 minutes or less. For sepsis, infuse at least $30 \mathrm{~mL} / \mathrm{kg}^{4}$ For routine maintenance, restrict the volume to $25-30 \mathrm{~mL} / \mathrm{kg} /$ day of water, and include $1 \mathrm{mmol} / \mathrm{kg} /$ day of potassium, sodium, and chloride along with 50-100 g/day of glucose to prevent starvation ketosis, though glucose should be avoided in most diabetic patients. With obesity, adjust the IVF to ideal body weight, and for patients who are older, frail, or admitted with renal or cardiac impairment, consider prescribing a lower range of fluid (20-25 $\mathrm{mL} / \mathrm{kg} /$ day). For redistribution or replacement, use sodium chloride or balanced crystalloids or consider colloids, which have a theoretical advantage in expanding intravascular volume while 
limiting interstitial edema. Note that colloids are more expensive, and definitive evidence supporting increased efficacy is lacking. Clinicians should monitor closely for hypovolemia, hypervolemia, and electrolyte abnormalities, particularly hypo- and hypernatremia that carry associated mental status implications and risk of central pontine myelinolysis. The inadvertent overuse of IVF is common in hospital settings, particularly when maintenance fluids are not discontinued upon patient improvement or when patients move between care areas. Thus, regular clinical reassessment of volume status is important.

\section{Recommendation 4}

In both noncritically ill and critically ill hospitalized patients, there is a benefit to using balanced crystalloids compared to isotonic saline in preventing major adverse kidney events and death. ${ }^{5,6}$

Two important studies in 2018 added new information to the existing NICE guidelines, addressing the previously unanswered question of the benefits of balanced crystalloids versus isotonic saline, one among non-critically ill patients and the other among critically ill patients. ${ }^{5,6}$ Prior data suggested that the use of isotonic saline is associated with multiple complications, including hyperchloremic metabolic acidosis, acute kidney injury, and death. In the non-critically ill population, the use of balanced crystalloids resulted in lower incidence of major adverse kidney events (absolute difference of $0.9 \%$ ), but did not change the number of hospital days (the primary outcome)..$^{5}$ In the critically ill population the use of balanced crystalloids resulted in lower rates of death, new renal replacement therapy, or persistent renal dysfunction, ${ }^{6}$ and the authors found preferential use of balanced crystalloids could prevent one out of every 94 patients admitted to the ICU from experiencing these adverse outcomes. Given the similar cost associated with isotonic saline and balanced crystalloids, these new findings suggest hospitalists should select balanced crystalloids if there is no compelling clinical reason to use isotonic saline.

\section{CRITIQUE}

While conflicts of interest are often a concern in clinical guidelines due to influence by pharmaceutical, device, and specialty interests, the United Kingdom's National Clinical Guideline Centre (NGC), which developed the NICE guidelines, is hosted by the Royal College of Physicians and has governance partnerships with the Royal College of Surgeons of England, Royal College of General Practitioners, and Royal College of Nursing. Each guideline produced by the NGC is overseen by an independent guideline committee comprised of healthcare professionals and patient representatives, and as a result, concern for conflicts of interest is low.

The NICE guidelines were created by a multidisciplinary team from multiple clinical specialties, and reviewed evidence addressing both clinical and health economic outcomes. Importantly, data from randomized controlled studies was relatively limited. The data excluded patients under 16 years of age, pregnant women, and those with severe liver or renal disease, diabetes or burns, as well as those in intensive care settings. Unfortunately, many medical patients cared for by hospitalists fall into one or more of these categories, limiting applicability of the guidelines.

Two important studies in 2018 added new information to the existing NICE guidelines, as outlined in Recommendation 4. ${ }^{5,6}$ Both of these studies occurred at a single institution, limiting their generalizability, though each study included a diverse patient population. In the ICU study, treating clinicians were aware of the composition of the assigned crystalloid so the decision to initiate renal-replacement therapy may have been susceptible to treatment bias. In addition, censoring of data collection at hospital discharge may have underestimated the true incidence of death at 30 days and overestimated persistent renal dysfunction at 30 days. Importantly, the trial design did not allow comparison of lactated Ringer's solution versus Plasma-Lyte. The non-ICU study evaluated patients who began treatment in the emergency department and were subsequently admitted to non-ICU inpatient units-a population that mirrors much of hospitalist practice, however the un-blinded design makes bias a concern. Finally, lactated Ringer's solution represented more than $95 \%$ of the balanced crystalloids used in the trial, so additional study is required to compare Plasma-Lyte with both saline and lactated Ringer's solution.

\section{AREAS IN NEED OF FUTURE STUDY}

More evidence is needed to better understand the appropriate use of IVF in specific clinical scenarios, including to determine if balanced solutions, as compared with isotonic saline, are superior across a spectrum of clinical conditions. For patients with an indication for maintenance fluid administration, determining if a higher sodium content reduces the risk of hyponatremia without increasing the risk of volume overload will help guide practice. Finally, more comprehensive study of the incidence of overuse and complications as a consequence of IVF, as well as the optimal frequency of lab monitoring, is needed to guide understanding of how practicing hospitalists and health systems can help reduce harm and waste

Disclosures: The authors have nothing to disclose.

\section{References}

1. Minto G, Mythen MG. Perioperative fluid management: science, art or random chaos? Br J Anaesth. 2015;114(5):717-221. doi: 10.1093/bja/aev067.

2. National Clinical Guideline Centre. Intravenous Fluid Therapy: Intravenous Fluid Therapy in Adults in Hospital, London: Royal College of Physicians (UK); 2013 Dec. Updated May 3, 2017. https://www.nice.org.uk/guidance/ cg174. Accessed January 25, 2019.

3. Hall A, Ayus J, Moritz M. Things we do for no reason: the default use of hypotonic maintenance intravenous fluids in pediatrics. J Hosp Med. 2018;13(9):637-640. doi: 10.12788/jhm.3040.

4. Rhodes A, Evans LE, Alhazzani W, et al. Surviving sepsis campaign: international guidelines for management of sepsis and septic shock 2016. Intensive Care Med. 2017;43(3):304-377. doi: 10.1007/s00134-017-4683-6.

5. Self WH, Semler MW, Wanderer JP, et al. Balanced crystalloids versus saline in noncritically ill adults. N Engl J Med. 2018;378(9):819-828. doi: 10.1056/ NEJMoa1711586.

6. Semler MW, Self WH, Rice TW. Balanced crystalloids versus saline in critically ill adults. N Engl J Med. 2018;378(9):829-839. doi: 10.1056/NEJMoa1711584. 